\title{
Photoreaction Dynamics of Full-length Phototropin from
}

\section{Chlamydomonas Reinhardtii}

Yusuke Nakasone ${ }^{\dagger}$, Masumi Ohshimat, Koji Okajima ${ }^{\dagger}$, Satoru Tokutomi ${ }^{\S}$, Masahide Terazima $^{\dagger *}$

†Department of Chemistry, Graduate School of Science, Kyoto University, Kyoto 6068502, Japan

Graduate School of Science and Technology, Keio University, Kanagawa 223-8522, Japan

$\S$ Department of Biological Science, Graduate School of Science, Osaka Prefecture University, Sakai, Osaka 599-8531, Japan

\section{SI-1. Absorption spectra and SDS-PAGE}
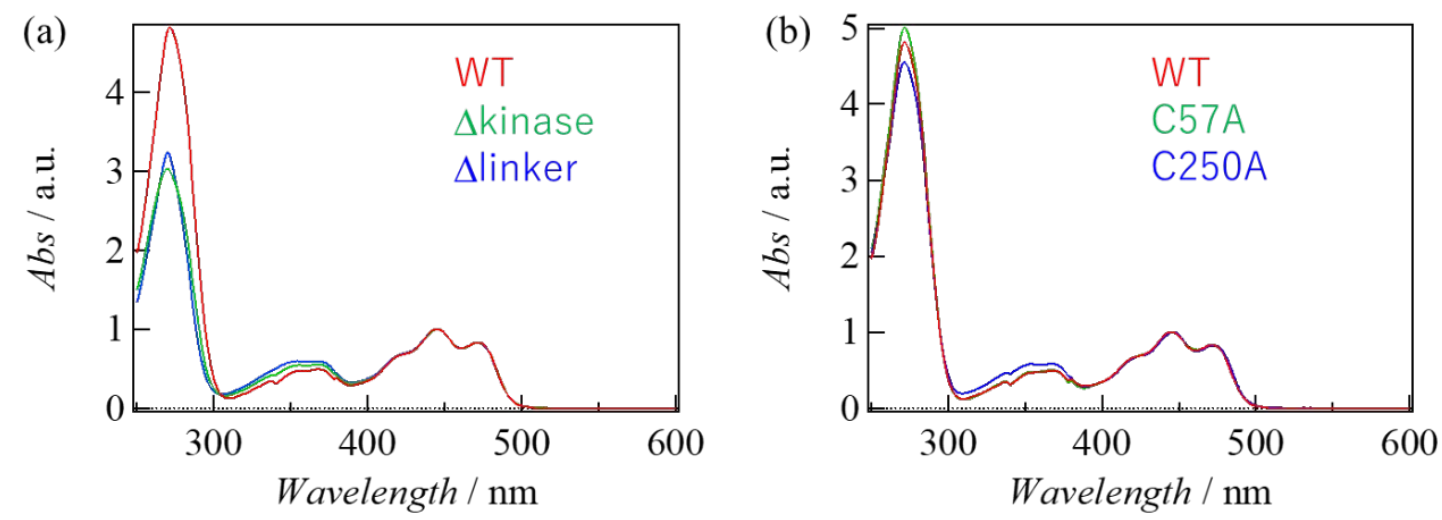

(c) $\begin{array}{llllllllllll}1 & 2 & 3 & 4 & 5 & 6 & 7 & 8 & 9 & 10 & 11 & 12\end{array}$

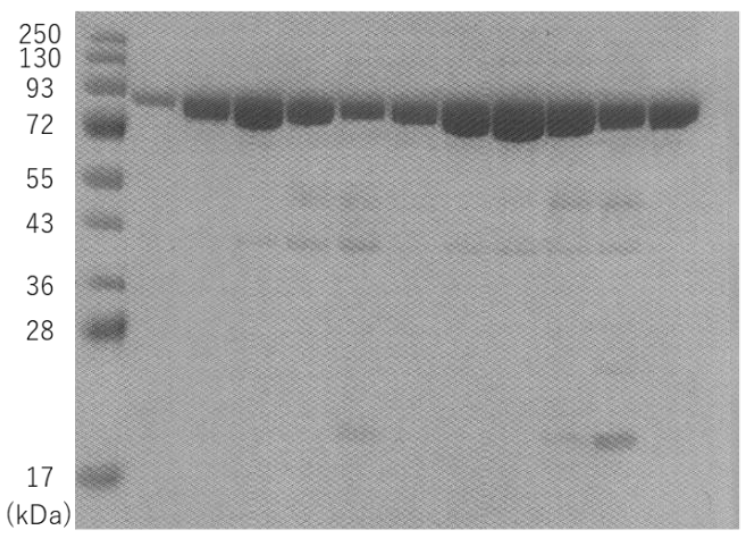

(d)
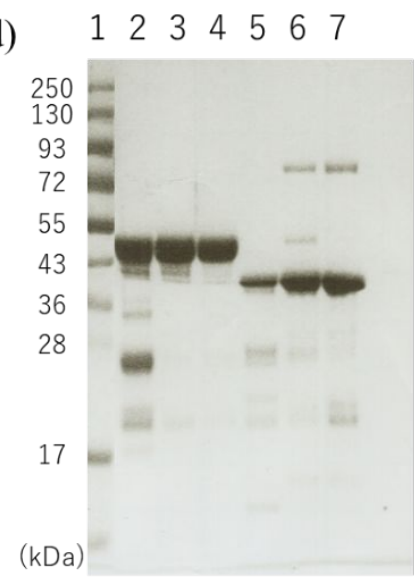
Figure S1. (a), (b) Absorption spectra of $\mathrm{Cr}$ phot and its derivatives. (c) SDS-PAGE of full-length $\mathrm{Cr}$ phot. Lane1: Marker, Lane2 6: several fractions of SEC of C57A mutant, Lane7 11: several fractions of SEC of C250A mutant, Lane12: wild type $\mathrm{Cr}$ phot. For TG and CD measurements, only Lane2 and 3 were used for C57A mutant and Lane7 and 8 were used for C250A mutant, because degradation products were slightly observed in other lanes. (d) SDS-PAGE of truncated variants of $\mathrm{Cr}$ phot. Lane1: Marker, Lane:2: $\Delta$ kinase, Lane3: C57A of $\Delta$ kinase, Lane4: C250A of $\Delta$ kinase, Lane5: $\Delta$ linker, Lane6: C57A of $\Delta$ linker, Lane 7: C250A of $\Delta$ linker. 


\section{SI-2. Photoreaction of C57A/C250A double mutant}

The TG signal of C57A/C250A double mutant is shown in Figure S2. The adduct formation signal observed within $10 \mu$ s completely disappeared, and instead, the slow decays of triplet states were observed with time constants of $28 \mu \mathrm{s}$ and $212 \mu \mathrm{s}$. These time constants are the same as those determined from C57A and C250A. Unexpectedly, the double mutant shows the diffusion signal in the time range of $300 \mathrm{~ms} \sim 3 \mathrm{~s}$ even though there is no adduct formation and the triple state decays completely within $1 \mathrm{~ms}$. This result implies that the double mutant does not recover to the initial state completely by the decay of the triplet state, and the photoproduct having a longer lifetime exhibits a $D$ change. From the sign of the refractive index change, the rise and decay components are assigned to the diffusion of reactant and product respectively, similar to the case of WT. We consider that this reaction is initiated by the photoinduced reduction of FMN. The intensity of diffusion peak, however, is much smaller than that of WT. This weak intensity indicates that the quantum yield of the reaction is extremely small. Due to this low quantum yield, the contribution of the CA mutants to the diffusion signal is negligible, and we do not consider this reaction for the analyses in the main text.

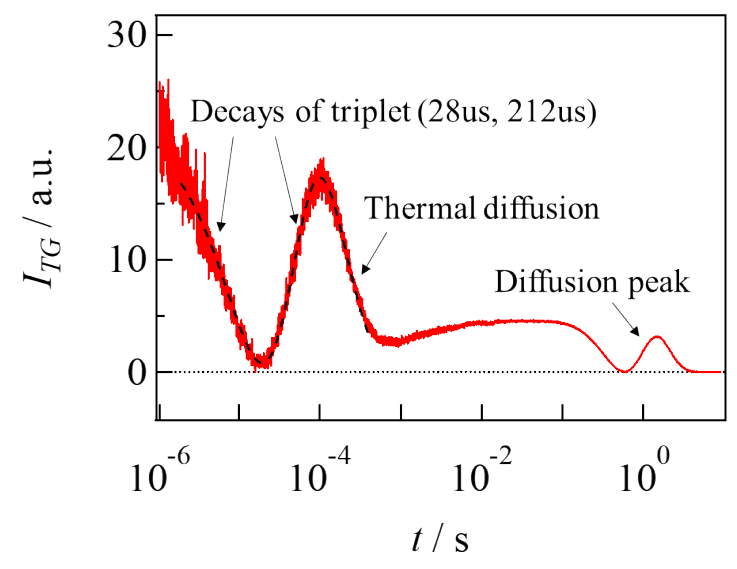

Figure S2. TG signal of C57A/C250A obtained at $\mathrm{q}^{2}=4.7 \times 10^{10} \mathrm{~m}^{-2}$. 
SI-3. Intensities of diffusion signals between CA mutants and WT $\mathrm{Cr}$ phot

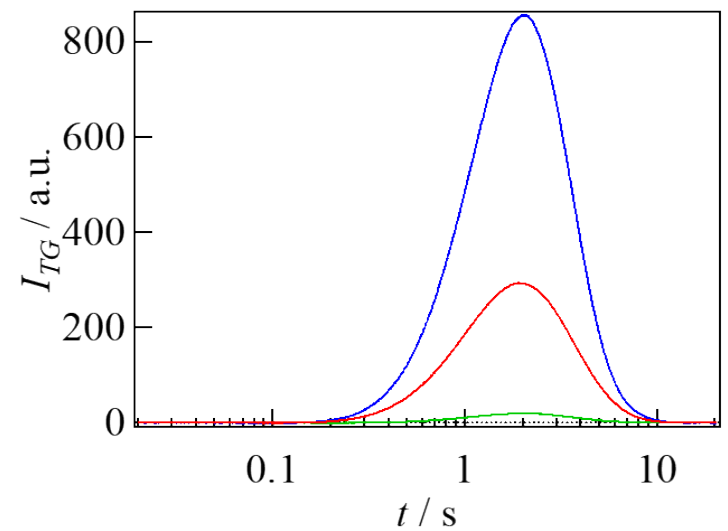

Figure S3. Diffusion signals of WT (red), C57A (green), and C250A (blue) obtained at $\mathrm{q}^{2}=1.8 \times 10^{10} \mathrm{~m}^{-2}$. The signals are normalized by the concentration of the adduct form. 


\section{SI-4. Oligomeric structure of $\boldsymbol{C r}$ phot}

The SEC profiles are shown in this section. Full-length $\mathrm{Cr}$ phot shows the elution peak at the molecular weight of $90 \mathrm{kDa}$ both under the dark and light conditions (Figure S4a). Though a slight increase of population of the dimer was observed in the SEC profile upon light illumination as a shoulder of the main peak, $\mathrm{Cr}$ phot mainly exists as a monomer regardless of the light condition. Dimerization is possibly induced by light, but its yield is negligibly small, and we exclude the dimerization reaction as the main cause of the $D$-change. The SEC profiles of $\Delta$ linker in the dark (blue) and light (red) states are shown in Figure S4b. The apparent molecular mass of $\Delta$ linker was determined to be 89 $\mathrm{kDa}$. This result indicates that $\Delta$ linker exists as the dimer (calculated molecular mass of $\Delta$ linker is $40 \mathrm{kDa}$ as a monomer). To confirm that the protein was in the light state for the SEC measurement, the absorbance at $450 \mathrm{~nm}$ was also monitored and shown in Figure S4c. The decrease of peak area upon light illumination showed that the light state was formed efficiently in the column. 


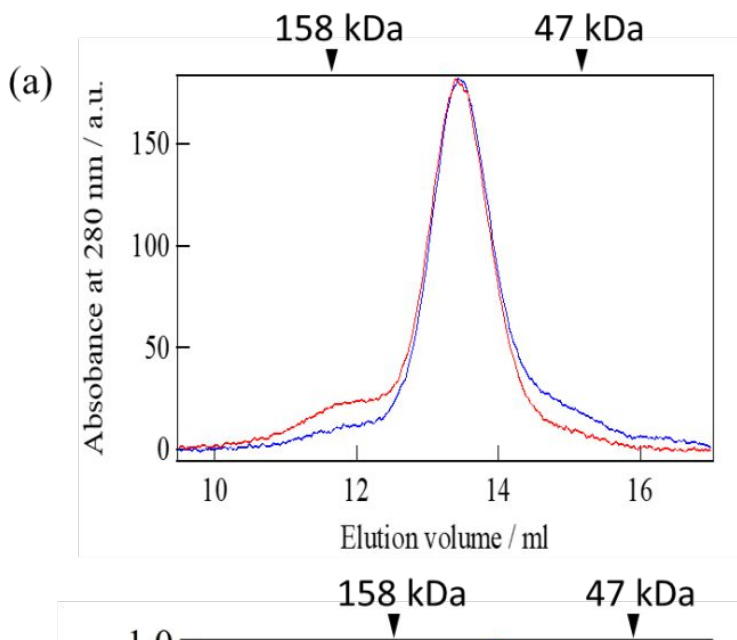

(b)

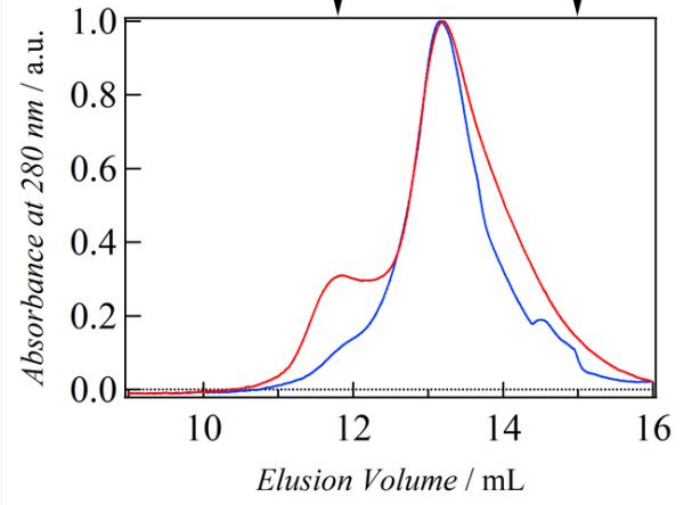

(c)

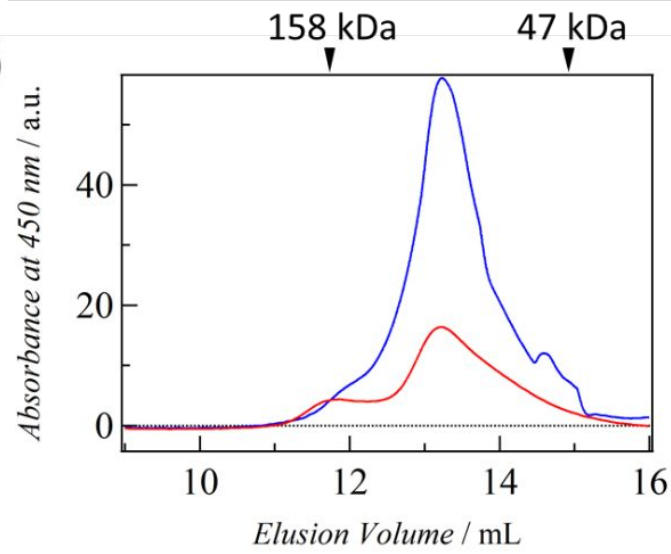

Figure S4. (a) SEC profiles of WT in the dark (blue) and light (red) states. The elution curves are normalized at peak intensity. (b) SEC profiles of $\triangle$ linker in the dark (blue) and light (red) states monitored at $280 \mathrm{~nm}$. The elution curves are normalized at peak intensity. (c) SEC profiles of $\Delta$ linker in the dark (blue) and light (red) states monitored at $450 \mathrm{~nm}$. 


\section{SI-5. Diffusion signals of truncated variants of C57A and C250A mutants.}
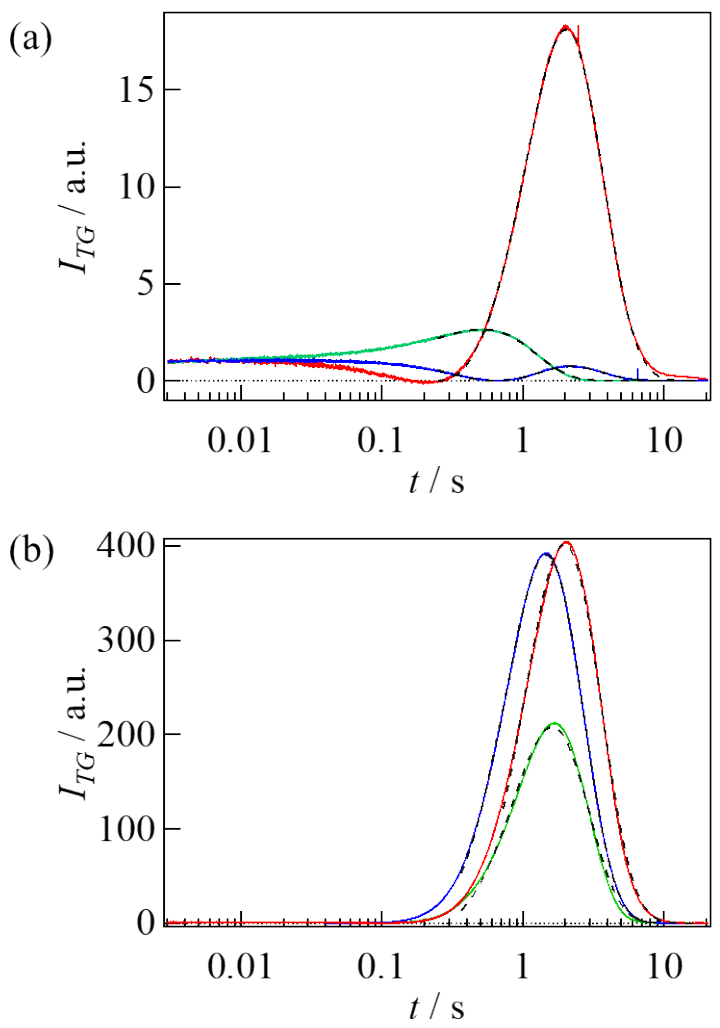

Figure S5. Diffusion signals of full-length $C r$-phot (red), $\Delta$ kinase (blue), and $\Delta$ linker (green) for (a) C57A and (b) C250A mutants obtained at $q^{2}=1.2 \times 10^{10} \mathrm{~m}^{-2}$. Concentrations of all samples are identical $(50 \mu \mathrm{M})$ and the signals are normalized by the concentration of the adduct form. The best fitted curves based on Eq. 2 are shown in black dotted lines. 